\title{
Anti-seismic characteristics of safety valve and its spring failure prediction analysis
}

\author{
Fanrong Meng, Huibing Zhang, Ye Dai ${ }^{*}$,Hanbo Zhang, Wenqiang Wei and Rui Liu \\ Harbin University of Science and Technology, School of Mechanical and Power Engineering, Heilongjiang Harbin 150080, China
}

\begin{abstract}
Safety valve is an important guarantee for nuclear power plant system. Its working environment is harsh, and medium is high-temperature corrosive. It is important to study the dynamic characteristics of nuclear safety valve under unsteady condition to improve the stability and safety of nuclear power plant piping system. Using the finite element method, mass simplification and Rayleigh method, the frequency response analysis of the safety valve and the spring is predicted to predict the seismic capacity under the earthquake load. Based on the verification of the dynamic characteristics of the valve body, the stress and strain analysis of the spring is carried out. To explore the failure condition of the safety valve spring, the lateral deviation linearity of the spring was tested and analyzed by setting up the offset test bench. The results show that the safety valve with the above analysis method can meet the working requirements under the shaking condition. The research results provide an important theoretical basis for the design and analysis of nuclear safety valves.
\end{abstract}

\section{Preface}

The safety valve is one of the most basic components in the nuclear power plant piping system and is mainly used to control the pressure, flow and flow of the fluid [1, 2]. Safety valve is an important guarantee for nuclear power plant safety system. Its working environment is harsh, and its medium has high temperature and corrosiveness. It is important to study the dynamic characteristics of nuclear safety valve under unsteady condition to improve the stability and safety of nuclear power plant piping system significance.

With the frequent occurrence of earthquake disasters, if the designed safety valve does not meet the seismic requirements, the earthquake will lead to nuclear valve nuclear leakage, which not only causes environmental pollution but also causes huge losses to human beings. Therefore, it is very necessary to analyze the seismic characteristics of the safety valve. Design safety valves not only for seismic analysis of safety valves, but also for failure prediction analysis of key spring components. This paper analyzes the spring failure prediction of key components of safety valves. The spring is a key component of the safety valve. The spring plays an important role in the stability and reliability of the safety valve. Due to the special working environment of the safety valve, the spring is required to have high temperature resistance and corrosion resistance as well as good fatigue resistance. Under the normal working condition of the safety valve, it is in a compressed state for a long time, so it is necessary to predict the failure before designing the spring, and then optimize the design. At present, spring-loaded safety valves are widely used in the industrial field, and both petrochemical and nuclear power plants play an important role. Spring functions mainly include storage energy, buffer pressure, control return, balance device and other functions [3-5]. The spring acts as the elastic part of the safety valve, and the calculation of the natural frequency of the safety valve spring is an important factor for the natural frequency of the safety valve [6-9].

In this paper, the safety valve will be studied and analyzed from the following two aspects: one is the seismic characteristics of the safety valve; the other is the spring failure prediction analysis. Perform frequency response analysis on the entire safety valve and key component springs, and stress and strain analysis on key spring components. By constructing a spring lateral offset test bench, the lateral deviation linearity of the spring in the safety valve is detected and analyzed. Analyze the experimental results and summarize the seismic capacity of the safety valve.

\section{Safety valve natural frequency analysis}

The research object of this paper is a spring direct load type safety valve. Its working principle uses the load to be applied to the valve disc. The main parts include valve body, valve seat, spring, valve flap, etc. The structure diagram is shown in Fig. 1. The valve twodimensional model is shown in Fig. 2.

* Corresponding author: daiye312@163.com 


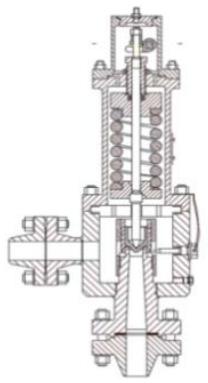

Fig. 1. Schematic diagram of safety valve structure.

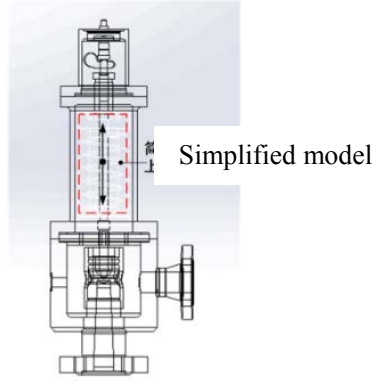

Fig. 2. Two-dimensional model of safety valve
The purpose of safety valve stress analysis is to verify its structure to meet the needs of production work. Apply a certain load on the valve body and other key components to analysis of the valve body deformation and stress conditions[2]. In the main parts of the safety valve, the allowable stress value of the valve body is the minimum[3]. Taking the valve body as an example, the structural stress analysis process is analyzed, and the other parts and components are analyzed emphatically.

\subsection{Establish a safety valve model}

The finite element and mass simplification method is used to analyze the seismic characteristics of the safety valve. The seismic performance of the safety valve is generally related to its own natural frequency. The firstorder natural frequency value of the designed safety valve is required to be greater than $33 \mathrm{~Hz}$. To improve the analysis efficiency of the safety valve under the premise of ensuring the calculation accuracy, the model can be simplified as appropriate, and the spring, the upper spring seat and the lower spring seat are regarded as a whole, and the removed mass is added to the valve seat, and the spring is applied to the upper and lower spring seats. The force can be seen as the force of a particle on the spring seat, which makes the calculated natural frequency value more accurate.

\subsection{Safety valve modal analysis pretreatment}

Create a three-dimensional model of the safety valve, including the valve body assembly, valve seat, valve flap and other structures.

The safety valve model does not include the spring, the upper spring seat, and the lower spring seat. Instead, the mass equivalent of this part of the removal is added to the adjustment screw to ensure that the overall quality of the safety valve remains unchanged. This not only reduces the analysis difficulty of the safety valve, but also improves the analysis efficiency of the safety valve.

\subsection{Safety valve natural frequency calculation result}

The whole safety valve is imported into the finite element in $\mathrm{x}$ - $\mathrm{t}$ format, and parameters are set, meshed and restrained. Finally, the first four-order modal analysis results of the safety valve are obtained, as shown in Fig. 3-Fig. 6.

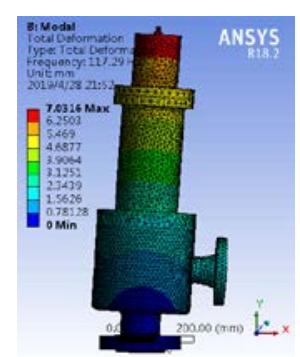

Fig.3. First-order mode shape

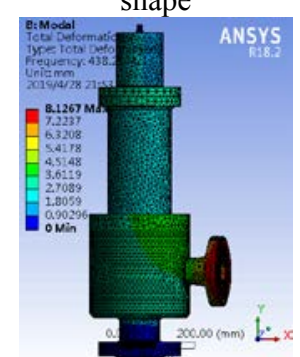

Fig.5. Third-order mode shape

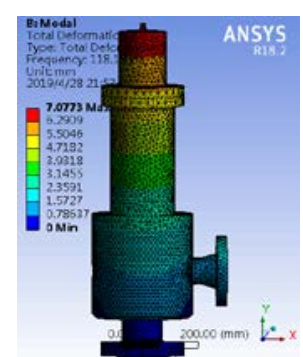

Fig.4. Second-order mode shape

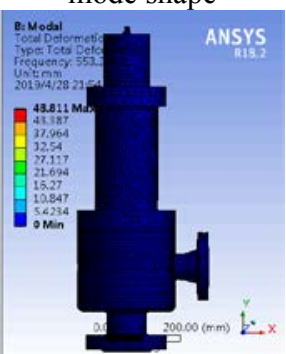

Fig.6. Fourth-order mode shape
It is shown in the above figures that the first-order mode shape of the safety valve belongs to the bending mode. From Fig. 3, the first-order natural frequency of the safety valve is $117.29 \mathrm{~Hz}$. The first-order natural frequency of the safety valve is designed to be greater than $33 \mathrm{~Hz}$, which meets the design conditions and therefore meets the safety requirements and valve seismic requirements.

\section{Calculation and analysis of natural frequency of spring}

In this paper, the Rayleigh method is used to calculate the kinetic energy of the spring's distributed mass into the total kinetic energy of the system, and the singledegree-of-freedom system is used to find the natural frequency [10]. A mathematical model is established for the spring, as shown in Fig. 7, assuming that each mass of the spring is fixed to one end of a straight elastic rod, and the other end is the same as the static deformation of each section under the axial force.

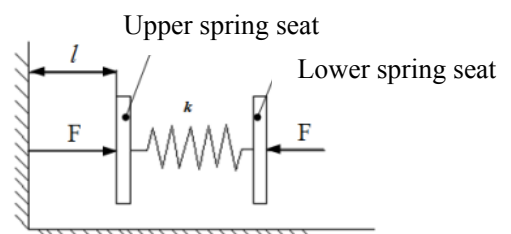

Fig.7. Spring Mathematical Model

According to Hooke's law, the deformation of each section of the spring is linearly related to the distance of the fixed end.

The kinetic energy of the entire system of the spring model is:

$$
T_{S}=\frac{1}{2} m_{e} x^{2}
$$

The displacement $\frac{\xi}{l} x$ from the left end is $\xi$, and the kinetic energy of the $d \xi$ spring is

$$
d T_{S}=\frac{1}{2} \frac{m_{s}}{l} d \zeta\left(\frac{\zeta}{l}\right)^{2}
$$

The total kinetic energy of the spring is 


$$
T_{S}=\int_{0}^{1} d T_{S}=\frac{1}{2} \frac{m_{S}}{3} x^{2}
$$

By combining the above formulas, you can get:

$$
m_{e}=\frac{1}{3} m_{s}
$$

It can be seen that the equivalent mass of the spring is one-third of the mass of the effective number of springs, and the total kinetic energy of the system is :

$$
\mathrm{T}=\frac{1}{2} m \dot{x}^{2}=\frac{1}{2}\left(m+\frac{m_{s}}{3}\right) \dot{x}^{2}
$$

The potential energy of the system is

$$
V=\frac{1}{2} k x^{2}
$$

Let $x=A \cos (w t-\varphi)$ be calculated from the conservation of energy: $T_{\max }=T_{\min }$

$$
\begin{array}{r}
\omega=\sqrt{\frac{k}{m+\frac{m_{S}}{3}}} \\
f=\frac{\omega}{2 \pi}=\frac{1}{2 \pi} \sqrt{\frac{k}{m+\frac{m_{S}}{3}}}
\end{array}
$$

Where: $m_{e}$ is the equivalent mass of the spring; $m_{-} \mathrm{s}$ is the mass of the effective number of springs; $m$ is the mass of the moving parts; for the safety valve, including the mass of the valve disc, the valve stem, the lower spring seat, and the number of invalid turns of the spring; The free length of the effective number of turns of the spring.

Modal analysis of the safety valve spring is carried out. The modal analysis is a numerical technique for calculating the vibration characteristics of the structure. The structural vibration characteristics include the natural frequency and the vibration mode [11-12].

In this paper, the first-order natural frequency value of the safety valve is obtained, and the result meets the seismic requirements. Then the natural frequency of the spring is analyzed to see if it meets the seismic requirements. The results are shown in Fig. 8-Fig. 11.

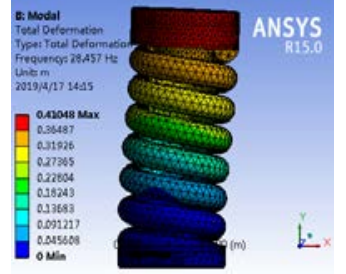

Fig.8. Spring first-order mode shape

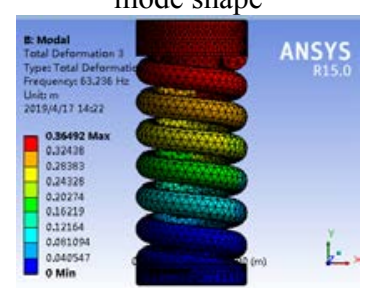

Fig. 10. Spring third-order mode shape

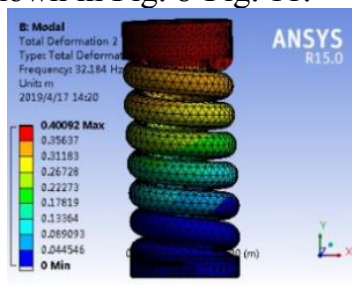

Fig.9. Spring second-order

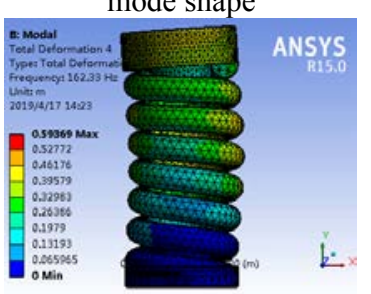

Fig. 11. Spring fourth-order mode shape mode shape

From the above simulation results, the following conclusions can be drawn: the first-order mode mode and the second-order mode shape of the spring and the fourth-order mode shape belong to the bending mode, and the spring array is orthogonal.

\section{Safety valve spring stress analysis}

\subsection{Spring strain analysis}

Due to the working principle of the safety valve, the two sides of the spring are subjected to the set pressure, and the spring is subjected to the axial pressure, assuming that the spring cross section is in the same plane as the spring axis. The internal forces of the spring section include the shear force Q and the torque MT, which are obtained according to the equilibrium relationship:

$$
\left\{\begin{array}{c}
Q=F \\
M_{T}=\frac{1}{2} F D
\end{array}\right.
$$

Where: $D$ is the diameter of the coil and $\mathrm{F}$ is the axial pressure to which the spring is subjected.

It is assumed that the shear stress caused by the shear force $\mathrm{Q}$ is evenly distributed in the cross section, as shown in Fig. 12, the shear stress caused by the shear force $\mathrm{Q}$ is :

$$
\tau_{Q}=\frac{4 F}{\pi d^{2}}
$$

According to the linear elastic circular axis torsion theory, the distribution of shear stress caused by the torque $\mathrm{T}$ in the linear elastic range is shown in the figure. The maximum shear stress is:

$$
\begin{aligned}
\tau_{\max } & =\frac{8 F D}{\pi d^{3}} \\
\frac{\tau_{Q}}{\tau_{\max }} & =\frac{d}{2 D}
\end{aligned}
$$

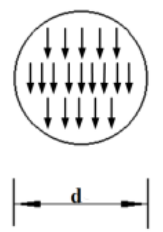

Fig. 12. spring shear stress distribution

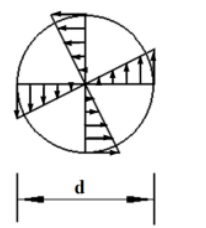

(1)

Fig. 13. Spring shear strain distribution

It can be obtained from the above formula that when the spring cross-sectional diameter $\mathrm{d}$ is much smaller than the average diameter $D$ of the coil, the shear strain distribution on the cross-section is negligible, and the strain distribution on the spring cross-section is as shown in Fig.12. and Fig.13. According to the principle of variable performance, the relationship between the axial compression amount $\lambda$ of the spring and the axial pressure $\mathrm{F}$ can be obtained by using the formula.

$$
\mathrm{F}=\frac{G d^{4}}{8 D^{3} N} \lambda
$$

Where $\mathrm{N}$ is the total number of turns of the spring. Through the above theoretical analysis, the shape variable and the shear stress are inversely proportional to the spring diameter under the axial or radial load of the safety valve spring.

\subsection{Spring strain analysis}

A mathematical model is established for the safety valve spring, the upper spring seat and the lower spring seat, and the stress and strain analysis is performed by using the finite element, and the results are shown in Fig. 14Fig. 17. 


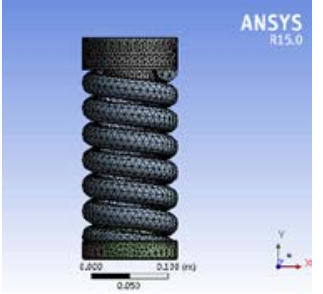

Fig. 14. Spring finite element model

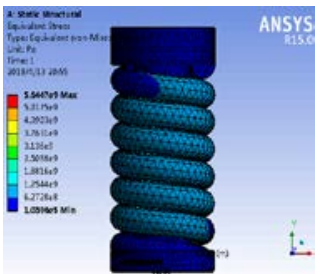

Fig. 16. Spring stress analysis results

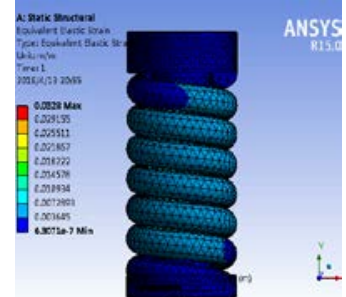

Fig. 15. Spring strain analysis results

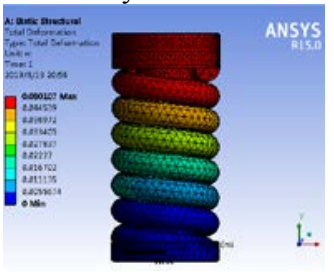

Fig. 17. Total spring deformation
From the simulation analysis results, it can be seen that the total deformation of the spring strain analysis is $0.05 \mathrm{~mm}$, and the stress analysis and strain analysis amount are relatively small, negligible, and meet the spring design requirements.

\section{Comparison of theoretical analysis and simulation analysis results}

Comparing the results of theoretical stress analysis and finite element stress analysis of spring, under the applied load, it can be seen that the amount of spring deformation is small and meets the requirements of seismic characteristics.

Comparing the natural frequency modal analysis and finite element analysis results of the spring, the interface definition safety valve spring setting material is analyzed on the finite element: the density is $7850 \mathrm{Kg} / \mathrm{m}^{3}$, and the elastic modulus is $2.0392 \times 10^{11} \mathrm{~Pa}$, Poisson's ratio is 0.33 . The result $f=30 \mathrm{~Hz}$ can be calculated from the above formula. The analysis result is $28.46 \mathrm{~Hz}$, and the theoretical calculation result is $30 \mathrm{~Hz}$. Deviation calculation:

$$
\delta=\frac{30-28.46}{30} \times 100 \%=4.6 \%
$$

\section{Safety valve spring lateral displacement experiment}

Considering the test requirements comprehensively, it is proposed to use the hammer method for modal test, the piezoelectric acceleration sensor is used for data acquisition of the safety valve, and the piezoelectric hammer is used for excitation. The one-way sensor measuring point layout is shown in Fig. 18, and three measuring points are arranged in each direction, for a total of six measuring points. Among them, measuring points 1 can test the natural frequency and bending mode of the $\mathrm{Y}$ direction, and the measuring points 2 can test the natural frequency and bending mode of the $\mathrm{X}$ direction. The two measuring points correspond to the first six channels of the dynamic signal processing system, and the third channel is the hammer channel.
The specific test scheme of artificial random excitation is: single input, multiple output, analysis frequency $500 \mathrm{~Hz}$, sampling frequency $1280 \mathrm{~Hz}$, points/frame: 8192 , collecting 20 frames of data, cumulative sampling time 128 seconds. Random tapping, hard disk acquisition, a total of 4 tests, respectively, different directions for different directions of excitation, of which one-way sensor layout program 2 times.

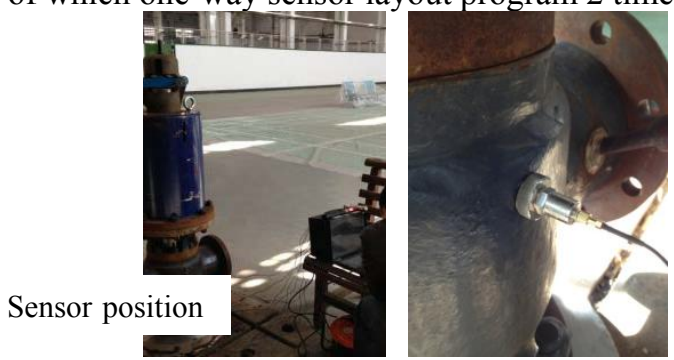

Fig. 18. Safety valve to be tested

The time domain curve measured by the hammer excitation at the position of the measuring point 3 is as shown in Fig. 19. According to the exciting direction, the natural frequency and the bending mode of the structure along the $\mathrm{Y}$ direction can be measured. According to the power spectral density function, the natural frequency of the structure along the $\mathrm{Y}$ direction can be identified. According to the imaginary part of the frequency response function (component analysis method), the natural frequency and the bending mode of the structure along the $\mathrm{Y}$ direction can be identified.

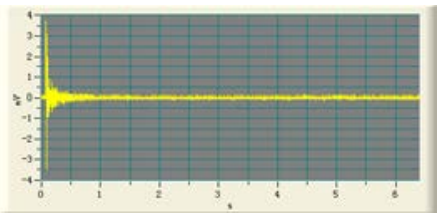

(a)

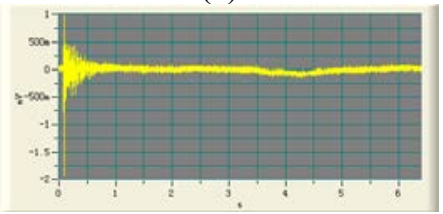

(b)

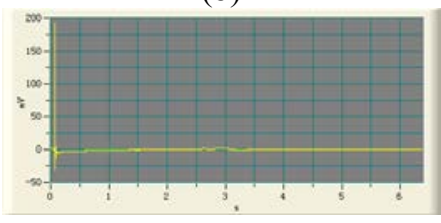

(c)

Fig. 19. Time domain waveform

The natural frequency of the safety valve measured in this test has little difference between different excitation modes and different modal parameter identification methods, and the results are more accurate. The Y-direction and the X-direction natural frequency are relatively close, mainly because the structure is a quasi-circumferential symmetrical structure. Compared with the finite element calculation results, the deviation is within $10 \%$, which is acceptable within the allowable range of engineering error. Comparing the corresponding mode shape results with the finite element results, the test test mode is consistent with the finite element calculation mode. 


\section{Conclusion}

1) Propose a method of quality simplification. The method ensures that the overall quality of the safety valve is unchanged, and the finite element analysis mode diagram is obtained, and the natural frequency value is greater than $33 \mathrm{~Hz}$, which satisfies the requirements of seismic working conditions.

2) The Rayleigh method is used to calculate the natural frequency of the spring. And combining theory and analysis to obtain the fourth-order natural frequency value of the spring, the first-order modal shape and the second-order modal shape and the fourth-order modal shape spring shape are obtained.

3) Perform stress and strain analysis on the key components of the spring to obtain the condition of failure of the safety valve spring. Under the axial or radial load, the deformation and shear stress are inversely proportional to the spring diameter.

The research is supported by National Natural Science Foundation of China (Grant No. E51505109), Natural Science Foundation of Heilongjiang Province of China (Grant No. LH2019E062), University Nursing Program for Young Scholars with Creative Talents in Heilongjiang Province (Grant No. UNPYSCT-2017077), Fundamental Research Foundation for Universities of Heilongjiang Province (LGYC2018JC040), China Postdoctoral Science Foundation (Grant No. 2016M591539), Heilongjiang Postdoctoral Science Foundation (Grant No. LBH-Z15102).

\section{References}

1. Chen Junjie, Yin Zhihong, He Jianghua, Shang Guanwen. Study on Modeling and Dynamic Characteristics of Air Spring System with Throttle Damping Hole and Additional Air Chamber[J]. Journal of Mechanical Engineering, 2017, 53(08): 166-174.

2. Godunov S K. Finite-difference method for numerical computation of discontinuous solutions of the equations of fluid dynamics [J]. 2017, 34:232235.

3. Chen Junjie, Yin Zhihong, He Jianghua, Shang Guanwen. Study on Modeling and Dynamic
Characteristics of Air Spring System with Throttle Damping Hole and Additional Air Chamber[J]. Journal of Mechanical Engineering, 2017, 53(08): 166-174.

4. Shen Wei, Zhang Qiangsheng, Chen Tianmin, et al. Seismic analysis and stress assessment of nucleargrade main steam safety valve[J]. Journal of Power Engineering, 2016, 36(2): 162-167.

5. Shen Wei, Zhang Qiangsheng, Chen Tianmin, et al. Seismic analysis and stress assessment of nucleargrade main steam safety valve[J]. Journal of Power Engineering, 2016, 36(2): 162-167.

6. Galbally D, García G, Hernando J, et al. Analysis of pressure oscillations and safety relief valve vibrations in the main steam system of a Boiling Water Reactor[J]. Nuclear Engineering \& Design, 2015, 293(3): 258-271 .

7. DAI Ye, LAI Yi-nan, ZHANG Yuan, REN Sheng-le, WANG Jian. A rapid simplification method based on rules and fuzzy judgments[J]. Journal of Harbin University of Science and Technology, 2013, 18(04): 37-41.

8. Li L Q, Ju N P, Zhang S, et al. Correction to: Seismic wave propagation characteristic and its effects on the failure of steep jointed anti-dip rock slope[J]. Landslides, 2018:1-2.

9. Dai Ye, Wu Nai-Qun*, Liu Rui, Meng Fan-Rong, Li Yuan. Integrated Design Method and Experimental Study for the Safety Performance Analysis of Nuclear Power Valve [J]. International Journal of Control and Automation, 2016, 9(10) : 201 208.

10. Song Yixin. Research on the Natural Frequency Analysis Method of ANSYS Safety Valve[J]. Science and Technology Innovation, 2017(26): 7778.

11. Zhou Bo, Wang Zhiyong, Xue Shifeng. Mechanical model of shape memory alloy superelastic coil spring[J/OL]. Journal of Mechanical Engineering: 19[2019-05-06].

12. Gao Hongxing, Chi Maoru, Zhu Wei, Liu Degang, Lin Jun. Study on Air Spring Model[J]. Chinese Journal of Mechanical Engineering, 2015, 51(04): 108-115. 